\section{Weiterbildung in Hausarztpraxen}

\author{
Aufbau und Durchführung des dreijährigen Pilotprojektes \\ 1. Juli 1998 - 30. Juni 2001
}

B. Rindlisbacher, M. Battaglia

Im Pilotprojekt «Weiterbildung in Hausarztpraxen (Praxisassistenz)» konnten in den drei vergangenen Jahren dank der ärzteeigenen Finanzierung 77 Assistenzen von im Durchschnitt knapp vier Monaten Dauer ermöglicht werden, 14 weitere erhielten einen fixen Beitrag. Jährlich hat das Projekt also rund 30 jungen Hausärzten zu einer strukturierten, wirklich praxisbezogenen Weiterbildungsphase in ihrem künftigen Berufsfeld verholfen. Die FMH, die Fachgesellschaften der Grundversorger und der VSA0 brachten dafür zusammen knapp 2 Mio. Franken auf, fast 1 Mio. wurde von den Lehrpraktikern beigesteuert und $700000 .-$ durch einen Lohnverzicht von den Assistenten getragen.

\section{Die Notwendigkeit}

Eine Berufsausbildung kann qualitativ nie befriedigend sein, wenn nicht ein Teil davon im Kontext der späteren beruflichen Tätigkeit stattfindet. Hausärzte müssen also einen Teil ihrer Weiterbildung in Hausarztpraxen absolvieren. Entsprechend ist die Praxisweiterbildung der Hausärzte in den meisten Ländern mit einem der Schweiz ähnlichen Standard des Gesundheitswesens längst selbstverständlich. In der EU schreibt die massgebende Richtlinie vor: "Die praktische Ausbildung (gemeint ist die Weiterbildung in Allgemeinmedizin) findet [...] während mindestens sechs Monaten in zugelassenen Allgemeinpraxen oder in zugelassenen Zentren für Erstbehandlung [Englisch: in an approved centre where doctors provide primary care] statt». Analoge Bestimmungen gelten für Kanada, Australien, die USA. Auch in der Schweiz ist die Notwendigkeit unter Fachleuten weitgehend unbestritten und wird durch die Evaluationsergebnisse dieses Projektes [1] weiter belegt. Ein Obligatorium ist bei uns nur deshalb noch nicht realisiert, weil die Finanzierung und damit auch die

Korrespondenz:

Dr. med. B. Rindlisbacher

Dr. med. M. Battaglia MPH

Co-Projektleiter

Sekretariat KHM

Effingerstrasse 40

CH-3008 Bern

$\begin{array}{ll}\text { KHM } & \text { Kollegium für Hausarztmedizin } \\ \text { SGAM } & \text { Schweizerische Gesellschaft für Allgemeinmedizin } \\ \text { SGIM } & \text { Schweizerische Gesellschaft für Innere Medizin } \\ \text { SGP } & \text { Schweizerische Gesellschaft für Pädiatrie } \\ \text { VSA0 } & \text { Verband Schweizerischer Assistenz- } \\ & \text { und Oberärztinnen/-ärzte } \\ \text { WB-Jahr } & \text { Weiterbildungsjahr }\end{array}$

logistischen Voraussetzungen (genügend Lehrpraxen, genügend ausgebildete Lehrpraktiker) bisher nicht befriedigend gelöst werden konnten.

\section{Vorgeschichte und Projektaufbau}

Die Forderung nach staatlicher Unterstützung einer bildungspolitischen Innovation, der Weiterbildung von Ärzten in privaten Hausarztpraxen, wurde 1994 von der Schweizerischen Sanitätsdirektorenkonferenz vorläufig zurückgestellt, sie verlangte zuerst eine Vorleistung der Ärzteschaft. Eine Arbeitsgruppe der FMH entwickelte im Herbst 1996 ein neues Finanzierungskonzept. Darauf basierend stellte das Kollegium für Hausarztmedizin (KHM) im Sommer 1997 der Ärztekammer den Antrag für ein vorerst dreijähriges "Pilotprojekt Praxisassistenz», welcher genehmigt wurde. Entgegen der ursprünglichen Absicht wurde dieses Pilotprojekt bisher allein von der Ärzteschaft finanziert mit einem jährlichen Beitrag von Fr. 20.- pro FMH-Mitglied und entsprechenden zusätzlichen Beiträgen von SGAM, SGIM, SGP und des VSAO. Weil die Mitglieder der Grundversorgergesellschaften SGAM, SGIM und SGP praktisch immer auch FMH-Mitglieder sind, bezahlten sie also einen zweifachen Beitrag. Nach den nötigen Aufbauarbeiten durch das KHM konnte im Sommer 1998 gestartet werden. Die Ärztekammer 2000 hat aufgrund der bis dahin gemachten Erfahrungen die Weiterführung des Projektes mit leicht veränderter Finanzierung für weitere zwei Jahre bis im Sommer 2003 sichergestellt.

Erfreulicherweise wurden eigene, kantonal unterstützte Praxisassistenzprojekte in den Kantonen Genf [2] und St. Gallen [3] aufgebaut.

\section{Die finanzielle Beteiligung vom Lehrpraktiker und Assistenten}

Die Lehrpraktiker wie auch die vom Projekt profitierenden Assistenten haben ebenfalls ihren Beitrag geleistet. Der von der Ärzteschaft finanzierte Projektfonds dient nur zum Auffüllen der Lücke zwischen dem Betrag, der von einem Lehrpraktiker als Lohnzahlung nebst seinem Engagement als Weiterbildner noch erwartet werden kann und dem, was einem Assistenten (oft verheiratet und mit Familie) als Minimallohn zugemutet werden kann. 
Der Assistentenlohn wurde gegenüber einem als Landesdurchschnitt berechneten Lohn im Spital um 25\% tiefer angesetzt, was zu Monatslöhnen in der Praxisassistenz von brutto zwischen Fr. 5460.(3. WB-Jahr) und 5960.- (5. WB-Jahr) führte. Die Reduktion wurde damit gerechtfertigt, dass der Assistent dafür die Garantie einer normalen Arbeitszeit von 43 bis maximal 50 Stunden pro Woche und damit optimale Rahmenbedingungen für Weiterbildung und Dienstleistung in der Praxis hat. Bei Vertretung erhält der Assistent 33\% mehr Lohn, was dann wiederum dem landesdurchschnittlichen Lohn im Spital entspricht.

Die pro Praxisassistenzmonat anfallenden Lohnkosten belaufen sich wegen der Arbeitgeberbeiträge an die Sozialversicherungen auf Fr. 6270.- bis Fr. 6850.-. Anfänglich hatte der Lehrpraktiker bei einer drei bis sechs Monate dauernden Praxisassistenz grundsätzlich 20\% dieser Lohnkosten zu tragen, 80\% wurden aus dem Projektfonds bezahlt. Alle Stellvertretungen von mindestens einem Tag wurden voll dem Lehrpraktiker in Rechnung gestellt.

Schon nach wenigen Monaten überstieg aber die Nachfrage die vorhandenen Mittel. So entschied sich das Aufsichtsgremium des Projektes, das Board, den Beitrag von 80\% der Lohnkosten nur noch während der minimal vorgeschriebenen Assistenzdauer von drei Monaten auszurichten. Etwa ein halbes Jahr später kam das Board auf diesen Entscheid zurück und in der Folge wurden bis zu drei weitere Monate immerhin noch zu 20\% aus dem Projektfonds unterstützt. Damit sollten die eher sinnvolleren, längeren Assistenzen wieder stärker gefördert werden. Vielfach wurden aber zusätzliche Monate auch voll durch den Lehrpraktiker bezahlt. Bei einer sechsmonatigen Praxisassistenz trug also der Lehrpraktiker mindestens 50\% der Kosten. Insgesamt wurden in den drei Jahren denn auch 45\% der effektiven Lohnkosten der Assistenten durch die Lehrpraktiker finanziert.

\section{Die Durchführung}

Die Durchführung des Projektes wurde dem Kollegium für Hausarztmedizin KHM übertragen, die Projektleitung einem Board (Leiter W. Ringli) mit Vertretern von FMH, SGAM, SGIM und SGP und des VSAO.

Es wurde eine zentrale Projektadministration eingerichtet mit den zwei Co-Projektleitern (B. Rindlisbacher und M. Battaglia) und dem KHM-Sekretariat. Diese ist für die operative Durchführung des Projektes verantwortlich. Damit wurden eine gute Kontrolle über die ausbezahlten Gelder, korrekte Lohnzahlungen an die Assistenten sowie ein umfassender Versicherungsschutz gewährleistet und gleichzeitig die Lehrpraktiker von administrativen Umtrieben stark entlastet. Über den Mediservice VSA0 wurden eigene Kollektivversicherungen eingerichtet. Der VSAO übernahm im Auftrag des KHM auch die Lohnbuchhaltung. Dass die Entlastung von den administrativen Umtrieben von den Lehrpraktikern geschätzt wurde, wird durch die Tatsache belegt, dass St. Gallen auf Wunsch der dortigen Lehrpraktiker auf unser System umschwenkte. Zwei Lehrpraktiker haben nur unsere Dienstleistung beansprucht, indem sie die Assistenz zwar vollständig selber finanzierten, diese aber administrativ über unser Büro abwickeln liessen.

\section{Einige statistische Daten}

Insgesamt standen in den drei Jahren knapp 2 Mio. Franken von FMH, VSA0 und Fachgesellschaften zur Verfügung (Tab. 1). Von den Lehrpraktikern wurde rund 1 Mio. Franken aufgebracht. Der Lohnverzicht der Assistenten machte rund einen Drittel des für die Gehälter aufgewendeten Betrages aus (rund Fr. 700 000.-; Tab. 2). Die Evaluation wurde hauptsächlich von der Schweizerischen Akademie der Medizinischen Wissenschaften mit einem Forschungsbeitrag von Fr. 120 000.- finanziert.

\section{Tabelle 1}

\begin{tabular}{llrr}
\hline Beiträge & & & $\%$ \\
Beitrag FMH & Fr. 1285000.00 & $41,9 \%$ \\
Beitrag SGAM & Fr. & 155000.00 & $5,0 \%$ \\
Beitrag SGIM & Fr. & 140000.00 & $4,6 \%$ \\
Beitrag SGP & Fr. & 35000.00 & $1,1 \%$ \\
Beitrag VSA0 & Fr. 340000.00 & $11,1 \%$ \\
Zahlungen Lehrpraktiker & Fr. 995000.00 & $32,4 \%$ \\
Beitrag SAMW Evaluation & Fr. 120000.00 & $3,9 \%$ \\
Total Beiträge & Fr. 3 070000.00 & $100,0 \%$ \\
\hline
\end{tabular}

\section{Tabelle 2}

\begin{tabular}{llr}
\hline Ausgaben & & \\
Lohnkosten Assistenten & Fr. 2175000.00 & $76,9 \%$ \\
Evaluation & Fr. $\quad 140000.00$ & $4,9 \%$ \\
Schulung Lehrpraktiker & Fr. $\quad 55000.00$ & $1,9 \%$ \\
Aufbau und Administration & Fr. 460000.00 & $16,3 \%$ \\
Total Aufwand & Fr. 2830000.00 & $100,0 \%$
\end{tabular}

$77 \%$ des ausbezahlten Geldes flossen in die Assistentengehälter, 16\% in Projektaufbau und -administration, 5\% in die Evaluation und 2\% in die Schulung der Lehrpraktiker.

In den drei Jahren wurden insgesamt 77 Assistenzen über das Projektbüro abgewickelt, 14 weitere erhielten einen fixen Beitrag, insbesondere in Zusammenarbeit mit dem Sankt-Galler Projekt. Von diesen 91 Assistenzen konnten 86 in die Evaluation einbezogen werden, 5 dauerten über das Stichdatum 30. Juni 2001 noch an und wurden deshalb nicht erfasst.

Jährlich hat das Projekt also rund 30 jungen Hausärzten zu einer Weiterbildungsphase in ihrem künftigen Berufsfeld verholfen. 70 Lehrpraktiker waren beteiligt. Insgesamt 95 haben in den drei Jahren den Einführungskurs (Hauptleitung D. Ackermann) absolviert. 
Die 77 direkt über das Projektbüro abgewickelten Assistenzen umfassten insgesamt 295 Monate (umgerechnet auf 100\%). 39 Assistenzen dauerten drei Monate bzw. bei Teilzeit entsprechend länger. Die übrigen dauerten umgerechnet auf 100\%-Tätigkeit bis zu 12 Monate (ab dem 7. Monat voll vom Lehrpraktiker finanziert). So ergab sich eine durchschnittliche Dauer von knapp vier Monaten zu 100\%. 245 Praxisassistenzmonate wurden zu 80\% bzw. 20\% mitfinanziert, die restlichen 50 von den Lehrpraktikern ganz allein bezahlt. Bei einem Viertel arbeitete der Assistent Teilzeit (zwischen 50 und 80\%).

Von den 75 ordentlich mitfinanzierten Assistenzen entfielen 51 (166 Monate) auf die Allgemeinmedizin, 14 (45 Monate) auf die Innere Medizin und 10 (34 Monate) auf die Pädiatrie. Die Innere Medizin war untervertreten, obschon diese Assistenzen aufgrund der Projektbestimmungen grundsätzlich bevorzugt wurden. Offenbar ist in diesem Fach das Interesse (noch) kleiner als bei Allgemeinmedizinern und Pädiatern.

Sieben Assistenzen von welschen Kollegen wurden in der französischen Schweiz durchgeführt, vier Assistenzen von italienischsprachigen im Tessin. Dazu kam je eine Assistenz eines französisch- und italienischsprachigen Assistenten in der Deutschschweiz.

\section{Ausblick}

Das von Anfang an deklarierte Ziel, für die Finanzierung des Projektes eine breitere Basis durch Beiträge von Kantonen, den Krankenkassen und allenfalls von der Pharmaindustrie und von Stiftungen zu erlangen, konnte bisher trotz intensiver Bemühungen (auch unter Mitarbeit eines professionellen found-raisers) nicht erreicht werden. Das Projekt musste zuerst aufgebaut und etabliert werden, bevor wir es möglichen Geldgebern präsentieren konnten. In den letzten zwei Jahren wurden nun verschiedene Kontakte aufgebaut. Das Projekt wird überall als gut und wichtig beurteilt, es wurde auch schon mehrfach Unterstützungsbereitschaft signalisiert, aber der Durchbruch ist noch ausgeblieben. $\mathrm{Ob}$ und wie rasch eine Mitfinanzierung von ausserhalb der Ärzteschaft erreicht werden kann, bleibt offen. Immerhin können die kantonal finanzierten Projekte in Genf und St. Gallen als richtungweisend hervorgehoben werden.

Im April 2002 muss die Ärztekammer entscheiden, ob sie im Sinne der Unterstützung der praxisbezogenen Weiterbildung weiterhin hinter dem Projekt steht und die weitere Finanzierung im bisherigen Umfang bewilligt.

\section{Literatur}

1 Schläppi P, Hofer D, Bloch R. Lernform Praxisassistenz bewährt sich. Schweiz Ärztezeitung 2002;83(9):410-6.

2 Brinkley B. Assistanat au cabinet médical: le modèle genevois. Primary Care 2001;1(15):454-5.

3 Schär HU, Jungi WF. St. Galler Praxisassistenz-Projekt 1997-2001, Primary Care 2002; 2 im Druck. 